\title{
8-year Analysis of the Prevalence of Lymph Nodes Metastasis, Oncologic and Pregnancy Outcomes in Apparent Early-Stage Malignant Ovarian Germ Cell Tumors
}

\author{
Usanee Chatchotikawong ${ }^{1}$, Irene Ruengkhachorn ${ }^{1 *}$, Chairat Leelaphatanadit ${ }^{1}$, \\ Nisarat Phithakwatchara ${ }^{2}$
}

\begin{abstract}
Purpose: To determine the rate of lymph node metastasis, oncologic and pregnancy outcomes in apparent early-stage malignant ovarian germ cell tumor (MOGCT). Materials and Methods: Medical records of apparent early-stage MOGCT patients undergoing primary surgical treatment at Siriraj Hospital, Bangkok, Thailand, between January 2006 and December 2013, were retrospectively reviewed. Results: Thirty-eight patients had apparent stage I-II MOGCT. The mean age was 22.1 \pm 7.7 years (median, 20.8 years; range, 7.7-35.6 years). The mean tumor size was $17.8 \pm 6.5 \mathrm{~cm}$ with a median of 20 (range 4-30) $\mathrm{cm}$. Three most common histopathologies were dysgerminoma ( 12 patients, $31.6 \%$ ), immature teratoma ( 12 patients, $31.6 \%$ ), and endodermal sinus tumor (6 patients, $15.8 \%$ ). Twenty-seven of 38 patients underwent lymphadenectomy; 13 patients $(48.2 \%)$ were stage IA and 8 patients $(29.6 \%)$ were stage IC. The rate of retroperitoneal nodes metastasis was $7.4 \%(2 / 27$ patients). At 26.1 months of median follow-up time (range 1.9-88.5 months), 9 patients retained fertility functions, with uneventful pregnancies in 3 of these. Only one patient $(2.6 \%)$ had progression of disease at 4.9 months after surgery. The 5-year survival rate was $97.4 \%$. Conclusion: As the rate of pelvic or para-aortic node metastasis in MOGCT is considerable, lymphadenectomy should be incorporated in surgical staging procedures.
\end{abstract}

Keywords: Ovary germ cell tumors - lymph node - lymphadenectomy - pregnancy outcome

Asian Pac J Cancer Prev, 16 (4), 1609-1613

\section{Introduction}

Ovarian germ cell malignancy mostly occurs in the second decades of life, accounting for $5 \%$ of ovarian malignancies, and is mainly unilateral, although 10$15 \%$ of dysgerminoma involve bilaterally (Gershenson, 2007; Bilici et al., 2014). Majority of this gynecologic malignancy has been very favorable outcomes, high chemosensitivity, and long-term survival. Successful surgery by fertility-preserving comprehensive approach, which means peritoneal cytology, meticulous pelvic and abdominal exploration, unilateral salpingo-ophorectomy, pelvic and para-aortic node dissection, omentectomy, and peritoneal biopsies to retain the uterus and contralateral normal ovary.

In clinical early-stage of malignant ovarian germ cell tumors (MOGCT) had lymph nodes metastasis rate of 10-18\% (Kumar et al., 2008; Mahdi et al., 2011; Kleppe et al., 2014). Lymph node metastasis is an independent predictor of poor survival in ovarian germ cell patients with a hazards ratio (HR) of 2.87 (95\%CI 1.439-5.725)
(Kumar et al., 2008). Whether ipsilateral or bilateral pelvic lymphadenectomy (LND) including para-aortic LND should be performed is still up for debate. Bilateral pelvic and para-aortic LND is a component of the standard surgery for evaluation of germ cell malignancy stage (Gershenson, 2007; Kleppe et al., 2014). However, it has been argued that a complete LND is not necessary and the para-aortic and bilateral pelvic lymph nodes should be sampled of only suspicious nodes or random sampling even in no suspicious area (Patterson et al., 2006). This procedure requires gynecologic oncology surgeon, moreover, operative time, blood loss, and risk of adjacent organs injury are higher than omitted. As the presence of lymph nodes metastasis can be used as a prognostic indicator of MOGCT, the decision of LND should be judged between the prevalence of nodal invasion and complications following the procedure.

Therefore the objective of this study was to determine the rate and pattern of lymph nodes metastasis in apparent early-stage MOGCT patients from our single tertiarycare center in order to provide the adequate treatment in 
Usanee Chatchotikawong et al

our population. The overall survival rate and pregnancy outcomes were also reported.

\section{Materials and Methods}

The study was approved by Siriraj Institutional Review Board. The design was retrospective review of medical records of patients who were MOGCT, disease confined in pelvis and underwent primary surgical treatment at Siriraj Hospital, Bangkok, Thailand, from January 2006 to December 2013.

The medical records of the studied women were reviewed including demographic data, surgical procedures, pathologic results, the International Federation of Gynecology and Obstetrics (FIGO) staging, treatment modalities, and oncologic outcomes. The operation considered a comprehensive fertility-preserving surgical staging including peritoneal cytology study, unilateral or bilateral salpingo-ophorectomy, pelvic and para-aortic node sampling or dissection, omentectomy, and peritoneal biopsies. The studied patients were categorized by surgical procedures into 2 groups; LND and non-LND groups regarding whether bilateral pelvic and para-aortic LND was included. The follow-up interval after initial treatment was every 3 months in the first 2 years, every 6 months in the third to fifth year, then annually. The attending doctor would evaluate clinical history, physical examination, and tumor markers in each visit. Survival duration was defined from the date of operation until the date of recurrence or progression (progression free survival, PFS) or death (overall survival, OS).

The data were analyzed using SPSS version 21.0 (IBM, Armonk, NY, USA). Descriptive statistics were used for the baseline characteristics, represented in number (percentage), or mean \pm standard deviation (SD), or median (interquartile range), as appropriate. The prevalence and site of lymph nodes spreading in LND group were determined. Pregnancy rate was estimated in patients who remain fertility function without contraception. Kaplan-Meier method was used for survival analysis of all studied patients.

\section{Results}

In this 8-years duration, of 48 patients received primary surgical treatment for MOGCT, 38 cases were apparent early-stage. Surgical staging procedure could not be completed in 11 cases by the reason of emergency operation $(n=1)$, operation during pregnancy $(n=1)$, undiagnosed ovarian malignancy during operation $(n=9)$. Twenty-seven patients had fertility-preserving comprehensive surgical staging, defined as a LND group. Para-aortic LND was performed only below inferior mesenteric artery (IMA) level in all patients.

Table 1 showed baseline characteristics and pathological reports of all 38 studied patients. The mean age of patients was $22.1 \pm 7.7$ years with a median of 20.8 (range 7.7-35.6) years. The mean body mass index (BMI) was $20.6 \pm 4.4 \mathrm{~kg} / \mathrm{m} 2$ with a median of 19.4 (range 11.4-33.3) kg/m2. The mean of tumor size was $17.8 \pm 6.5 \mathrm{~cm}$ with a median of 20 (range $4-30) \mathrm{cm}$. The histopathologic features were dysgerminoma (12 patients, $31.6 \%$ ), immature teratoma, (12 patients, $31.6 \%)$, mixed germ cell tumors ( 7 patients, $18.4 \%$ ), endodermal sinus tumor (6 patients, $15.8 \%$ ), and choriocarcinoma (1 patient, $2.6 \%$ ). Of 27 patients who underwent LND, 13 patients $(48.2 \%)$ were stage IA, 1 patient $(3.7 \%)$ were stage IB, 8 patients $(29.6 \%)$ were stage IC, 2 patients $(7.4 \%)$ were stage IIC, 1 patient $(3.7 \%)$ in stage IIIA, and 2 patients $(7.4 \%)$ in stage IIIC.

Hysterectomy was done in 4 patients who had completed their childbearing. The majority of cases had unilateral ovarian involvement, confined to right and left ovary in 14 and 9 cases, respectively. Three patients underwent bilateral salpingo-oophorectomy (bilateral ovarian tumors, $\mathrm{n}=1$; co-existing gonadal dysgenesis, $\mathrm{n}=1$; and an operation performed by non-gynecologic oncologist, $n=1$ ). Contralateral ovarian cystectomy was done in two cases, one with endodermal sinus tumor and in the other with immature teratoma. There was one patient (2.6\%) with microscopic omentum metastasis. Among 20 patients neither with extraovarian spread nor with ruptured tumors, 4 patients (20\%) had positive cytology.

The mean number of nodes removal was $16.1 \pm 11.8$ nodes with a median of 16 (range 2-36) nodes. When considering the area of node removal, lymph node retrieval ranged 1-18 nodes for right pelvic area, 1-19 nodes for left pelvic area, and 1-14 nodes for para-aortic area. Of 27 LND patients, 2 cases (7.4\%) had lymph nodes metastasis. One patient had only ipsilateral pelvic node metastasis and

Table 1. Patients Characteristics and Histopathologic Results of 38 Studied Patients

\begin{tabular}{lrr}
\hline Characteristics & $\begin{array}{c}\text { LND }(\mathrm{n}=27) \\
\text { Mean } \pm \text { SD or } \mathrm{n}(\%)\end{array}$ & $\begin{array}{c}\text { non-LND }(\mathrm{n}=11) \\
\text { Mean } \pm \text { SD or } \mathrm{n}(\%)\end{array}$ \\
\hline Age $^{\mathrm{a}}$ (years) & $21.4 \pm 7.7,19.6$ & $23.9 \pm 7.5,25.5$ \\
& $(7.7-33.7)$ & $(13.3-35.6)$ \\
Body mass index $^{\mathrm{a}}$ & $20.0 \pm 4.1,20.0$ & $22.2 \pm 4.8,22.2$ \\
$\left(\mathrm{~kg} / \mathrm{m}^{2}\right)$ & $(11.4-31.2)$ & $(16.8-33.3)$ \\
Tumor size $^{\mathrm{a}}(\mathrm{cm})$ & $17.2 \pm 5.8,20.0$ & $19.2 \pm 8.2,18.0$ \\
\multicolumn{4}{c}{$(4.0-28.0)$} & $(6.0-30.0)$ \\
Postmenarche & $17(62.9)$ & $2(18.2)$ \\
Presenting symptoms & & \\
Palpable mass & $11(40.8)$ & $2(18.2)$ \\
Abdominal distension & $9(33.3)$ & $1(9.1)$ \\
Pelvic pain & $4(14.8)$ & $6(54.5)$ \\
Other & $3(11.1)$ & $2(18.2)$ \\
Histopathology & & \\
Dysgerminoma & $10(37.1)$ & $2(18.2)$ \\
Immature teratoma & $7(25.9)$ & $5(45.4)$ \\
Endodermal sinus tumor & $5(18.5)$ & $1(9.1)$ \\
Choriocarcinoma & 0 & $1(9.1)$ \\
Mixed germ cell tumors & $5(18.5)$ & $2(18.2)$ \\
FIGO stage (n=27) & & \\
IA & $13(48.2)$ & \\
IB & $1(3.7)$ & \\
IC & $8(29.6)$ & \\
IIB & 0 & \\
IIC & $2(7.4)$ & \\
IIIA & $1(3.7)$ & \\
IIIC & $2(7.4)$ & \\
\hline
\end{tabular}

${ }^{a}$ report as mean \pm standard deviation, median (range); FIGO, the International Federation of Gynecology and Obstetrics; LND, lymphadenectomy 
Table 2. Characteristic of two Patients who had Lymph Node Metastasis from 27 Patients in the Lymphadenectomy Group

\begin{tabular}{llcllcc}
\hline Age (years) & Symptoms & Side of ovary, size of tumors & LN metastasis & Histopathology & OS (months) & Outcome \\
\hline 27 & Pelvic pain & Right, $4 \mathrm{~cm}$ & Right PLN & Dysgerminoma & 12.9 & AWOD \\
16 & Pelvic mass & Right, $20 \mathrm{~cm}$ & PAN & Dysgerminoma & 5.7 & AWOD \\
\hline
\end{tabular}

AWOD, alive without disease; FIGO, the International Federation of Gynecology and Obstetrics; LN, lymph node; OS, overall survival; PAN, para-aortic node; PLN, pelvic lymph node

Table 3. Prevalence of Retroperitoneal Node Metastasis in Apparent Early-Stage Malignant Ovarian Germ Cell Tumors among those with Comprehensive Lymph Node Staging

\begin{tabular}{|c|c|c|c|c|c|c|}
\hline \multirow[t]{2}{*}{ Authors } & \multirow{2}{*}{$\begin{array}{l}\text { number of LND/ } \\
\text { total GCT cases }\end{array}$} & \multirow{2}{*}{$\begin{array}{c}\text { Mean, Median (range) } \\
\text { of node retrieval }\end{array}$} & \multirow[t]{2}{*}{ Positive nodes $\mathrm{n}(\%)$} & \multicolumn{3}{|c|}{ Site of lymph nodes metastasis $n(\%)$} \\
\hline & & & & PLN & PAN & Both \\
\hline Liu et al. 2013 & $16 / 30$ & ns & 0 & ns & ns & ns \\
\hline Mahdi et al. 2011 & $493 / 1083$ & $\begin{array}{c}11^{\mathrm{a}} \text { and } 2^{\mathrm{b}} \\
8^{\mathrm{a}} \text { and } 1^{\mathrm{b}} \\
\quad(1-47)\end{array}$ & $52(10.5)$ & ns & ns & ns \\
\hline Kumar et al. 2008 & $432 / \mathrm{ns}$ & $\begin{array}{c}12^{\mathrm{a}} \text { and } 14^{\mathrm{b}} \text {, } \\
\text { ns, } \\
(1-96)\end{array}$ & $51(11.8)$ & ns & ns & ns \\
\hline Rogers et al. 2004 & $21 / 57$ & ns & 0 & ns & ns & ns \\
\hline This study & $27 / 38$ & $\begin{array}{c}16.1, \\
16, \\
(2-36)\end{array}$ & $2(7.4)$ & $1^{\mathrm{c}}(50.0)$ & $1(50.0)$ & 0 \\
\hline
\end{tabular}

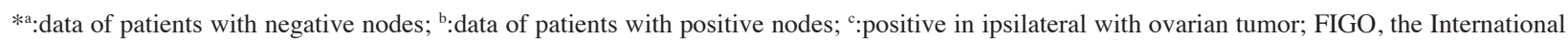
Federation of Gynecology and Obstetrics; LND, lymphadenectomy; ns, not specified; PAN, para-aortic node; PLN, pelvic lymph node

the other had isolated para-aortic node metastasis (Table 2). Prevalence and pattern of lymph nodes metastasis in malignant germ cell tumors of ovary from literatures review was showed in Table 3.

Thirty-two patients $(84.2 \%)$ received adjuvant chemotherapy, which is 22 in LND group and 10 in nonLND group. Bleomycin, etoposide and platinum (BEP) regimen was given to almost all patients $(31 / 32,96.9 \%)$. Only one patient received platinum plus etoposide. Median follow-up time was 26.1 (range 1.9-88.5) months with a mean of $35.5 \pm 26.2$ months. The overall 5-year survival rate was $97.4 \%$. Disease progression during primary treatment was reported in one LND patient (1 in 38 patients, $2.6 \%$ ) with stage IA immature teratoma, developed at 4.9 months after surgery, and unfortunately she passed away. The rate of loss to follow-up was $18.4 \%$ (7 in 38 patients), 5 in LND group and 2 in non-LND group. Among those with fertility preservation, 9 patients attempted pregnancy and only 3 patients had uneventful term pregnancies.

\section{Discussion}

MOGCT is rapid growing tumors, fortunately, usually affects unilateral ovary, common in healthy girl or young women, and responses well to chemotherapy. Fertility-sparing comprehensive surgical staging is safe (Gershenson, 2007; Colombo et al., 2012; Low et al., 2012). Even though the principle of completely bilateral pelvic and para-aortic LND for surgical staging in epithelial ovarian carcinoma has been accepted, but in fascinated neoplasm such MOGCT has not been well studied. Despite the current evidence has convinced the essential of LND in MOGCT staging, it remains controversial whether it results in the better oncologic outcomes. Owing to its rarity, accrual of a large number of MOGCT samples is difficult to achieve, therefore affirming evidence is restraint. This study reported 8-years' experience of a single medical university hospital in Thailand.

In the current study, the retroperitoneal nodal metastasis rate was $7.4 \%$, which slightly lower than previous studies from United State, 10.5-11.8\% (Kumar et al., 2008; Mahdi et al., 2011) but higher than a study from China, zero (Liu et al., 2013). These seemingly conflicting results may be explained by several hypotheses. Firstly, the potentiality of lymphatic spreading is presumably associated with some biological, social or environmental factors, expressed by racial or genetic diversity. Secondly, the number and level of LND also influenced the prevalence of lymph nodes metastasis. Logically, there is a greater probability of microscopic nodal metastasis detection in case of higher number of retrieved nodes or complete LND in both bilateral pelvic and para-aortic regions. Thirdly, the more proportion of dysgerminoma patients is, the higher rate of lymph nodes involvement is. In this study, dysgeminoma was a common pathology (31.6\%) and 2 of 10 dysgerminoma patients $(20 \%)$ who underwent LND had nodal spreading. This is comparable to the study of 1083 patients with clinical stage I MOGCT and 493 underwent LND, they found the rate of lymph nodes metastasis was $10.5 \%$ and highest in dysgerminoma patients, 18\% (Mahdi et al., 2011). Furthermore, a study of the Surveillance, Epidemiology, and End Results Program (SEER) revealed that dysgeminoma had the highest rate of nodal metastasis (28\%) of any other MOGCTs (Kumar et al., 2008).

As a high rate of lymph nodes metastasis in clinical early-stage dysgerminoma tumor, so recognition of this type of MOGCT required for surgical plan during surgeon facing it. The methods to predict dysgerminoma 
are as follows: $(i)$ solid ovarian tumors in young women; (ii) preoperative rising lactate dehydrogenase ( $\mathrm{LDH})$, these findings should refer to gynecologic oncologists; (iii) intraoperative suspicion by open cut surface of specimen; and (iv) histopathology of frozen section. When dysgerminoma is diagnosed, comprehensive LND should be performed immediately. However, the authors suggest that all solid ovarian tumors in young women should be operated by gynecologic oncologists, which would achieve accurate staging and optimal debulking of tumors in advanced cases to ensure a high chance of cure for these patients.

We attempted to localized location of nodal metastasis. Patterns of metastasis such those described in the present study reassure us that ipsilateral pelvic LND is insufficient for surgical staging of apparent early-stage MOGCT. Consistent with the fact that lymphatic drainage of ovary can get through para-aortic area as primary nodal station, and these findings consolidate the current evidence to enhance systematic bilateral pelvic and para-aortic LND in staging procedures of MOGCT. Moreover, bilateral pelvic and para-aortic LND can inform the accurate extension and prognosis of the disease, elimination of some microscopic tumors, being helpful for further treatment after surgery especially the number of cycles of chemotherapy, and inform the prognosis.

The overall 5-year survival rate of the current study was fabulous, $97.4 \%$. A study in patients who had disease grossly confined to the ovary reported a rate of 93.4-97.7\% (Mahdi et al., 2011). A report of stage I-IV MOGCT with BEP for 3 or 4 cycles had an overall 5-year survival of $96 \%$, and when focused on dysgerminoma or early-stage disease this rate was 100\% (Dimopoulos et al., 2004). A report of Thai patients with stage I-IV MOGCT had an 10-years PFS and OS of $81.9 \%$ and $86.2 \%$, respectively (Neeyalavira and Suprasert, 2014). The pregnancy rate $(33.3 \%)$ in this study population is quite lower than other previous studies, 45.9-50\% (Patterson and Rustin, 2008; Liu et al., 2013). This might be affected from retrospective design of studies, the racial difference and the small sample size of this study.

The high rate of loss to follow-up patients is a problem in Thai populations. Because it is a disease affecting girls or young women, it has a high cure rate following primary treatment, with a low relapse rate and a long period of survival. Young patients often believe in their good health and do not realize the importance of regular follow-up for life. This might confound the median follow-up time of our study. It is quite a challenge for the surveillance and quality of life assessment of this enchanting MOGCT.

It is hard to identify prognostic factor for recurrent disease or death in this study due to a small sample size and quite low number of events. A previous study stated that non-dysgerminoma and advanced FIGO stage were significant prognostic factors for treatment failure and short survival (Lai et al., 2005). Another study suggested that higher FIGO stage and rising tumor markers were predictors of poor survival with the HR of 5.96 (95\% CI 3.47-10.2) and HR of 3.9 (95\%CI 1.40-10.9), respectively (Murugaesu et al., 2006). Conversely, for disease confined to the ovaries, a multivariate analysis from SEER data revealed that neither LND nor nodal metastasis was prognostic factor with the HR of 1.26 (95\%CI 0.62$2.58)$ and HR of 2.7 (95\%CI 0.67-10.96), respectively (Mahdi et al., 2011). Another point of view, relapse rate, mortality, overall survival and tumor-free survival at 5 years, between comprehensive surgical staging procedures with unilateral salpingo-ophorectomy removal were comparable (Liu et al., 2013). To date, data of prognostic factors of apparent early-stage of MOGCT is scanty and might be difficult to resolve because it is an uncommon disease.

This is the first study reporting the prevalence of node-positive in early-stage MOGCT in our population, which provides the information applicable in our clinical practice. Nevertheless, the retrospective design and small sample size of this study limit the result analysis regarding the prognostic factor of recurrence or death and the advantage of LND on overall survival. These warrant further multicenter, prospectively well-designed study, rather than clinical and pathological information, study using genomic model will be necessary to triage patients in order to identify the candidate of LND, the optimal chemotherapeutic agents, and cost-effective individual surveillance.

The authors conclude that high rate of retroperitoneal nodes metastasis in early-stage MOGCT, highlights the necessity of pelvic and para-aortic LND in surgical staging.

\section{Acknowledgements}

This research was supported by Siriraj Research Development Fund.

\section{References}

Bilici A, Inanc M, Ulas A, et al (2014). Clinical and pathologic features of patients with rare ovarian tumors: multi-center review of 167 patients by the Anatolian Society of Medical Oncology. Asian Pac J Cancer Prev, 15, 6493-9.

Colombo N, Peiretti M, Garbi A, et al (2012). Non-epithelial ovarian cancer: ESMO Clinical practice guidelines for diagnosis, treatment and follow-up. Ann Oncol, 23, 20-6.

Dimopoulos MA, Papadimitriou C, Hamilos G, et al (2004). Treatment of ovarian germ cell tumors with a 3-day bleomycin, etoposide, and cisplatin regimen: a prospective multicenter study. Gynecol Oncol, 95, 695-700.

Gershenson DM (2007). Management of ovarian germ cell tumors. J Clin Oncol, 25, 2938-43.

Kleppe M, Amkreutz LC, Van Gorp T, et al (2014). Lymph-node metastasis in stage I and II sex cord stromal and malignant germ cell tumours of the ovary: a systematic review. Gynecol Oncol, 133, 124-7.

Kumar S, Shah JP, Bryant CS, et al (2008). The prevalence and prognostic impact of lymph node metastasis in malignant germ cell tumors of the ovary. Gynecol Oncol, 110, 125-32.

Lai CH, Chang TC, Hsueh S, et al (2005). Outcome and prognostic factors in ovarian germ cell malignancies. Gynecol Oncol, 96, 784-91.

Low JJ, Ilancheran A, Ng JS (2012). Malignant ovarian germ-cell tumours. Best Pract Res Clin Obstet Gynaecol, 26, 347-55.

Liu Q, Ding X, Yang J, et al (2013). The significance of comprehensive staging surgery in malignant ovarian germ 
cell tumors. Gynecol Oncol, 131, 551-4.

Mahdi H, Swensen RE, Hanna R, et al (2011). Prognostic impact of lymphadenectomy in clinically early stage malignant germ cell tumour of the ovary. Br J Cancer, 9, 493-7.

Murugaesu N, Schmid P, Dancey G, et al (2006). Malignant ovarian germ cell tumors: identification of novel prognostic markers and long-term outcome after multimodality treatment. J Clin Oncol, 24, 4862-6.

Neeyalavira V, Suprasert P (2014). Outcomes of malignant treated in Chiang Mai University Hospital over a nine year period. Asian Pac J Cancer Prev, 15, 4909-13.

Patterson DM, Rustin GJ (2006). Controversies in the management of germ cell tumours of the ovary. Curr Opin Oncol, 18, 500-6.

Patterson DM, Murugaesu N, Holden L, Seckl MJ, Rustin GJ (2008). A review of the close surveillance policy for stage I female germ cell tumors of the ovary and other sites. Int $J$ Gynecol Cancer, 18, 43-50. 